\section{Uso de herramientas de la Web 2.0 en la metodología aula invertida: una opción para clases a distancia en tiempos de COVID-19}

\author{
CARLOS FLORES-ANGULO ${ }^{1,2, a}$, JOSÉ CALLEJA ${ }^{2, b}$, \\ PAULO SANDOVAL ${ }^{3, c}$
}

\section{Use of web 2.0 Tools in the flipped classroom methodology: an option for distance learning}

Background: The flipped classroom is an active method where information in delivered before class and the time allocated for it is dedicated to achieving more complex learning objectives. Diverse resources are used in each phase of the methodology. However, Web 2.0 or "Social Web" tools allow greater personal and remote interaction. Aim: To describe the perception about flipped classroom methodology implemented using web 2.0 tools in 48 medical students from the University Mayor. Material and Methods: The students were invited to view four video-classes available on Edpuzzl. During the activity, questions about the topic were inserted to promote understanding, research, and teamwork, then the topic was discussed using Case-Based Learning through the Quizizz program. A survey composed of 12 closed items and an open question was applied to analyze the perceptions of students. Data were analyzed using the SPSS and Atlas-Ti programs. Results: A high satisfaction rate was observed about the way in which the content, discussion and communication of the information was presented. The methodology encouraged the development of generic competences, but it was not always positively perceived. Conclusions: The flipped classroom methodology developed with Edpuzzle was very well evaluated by students. This teaching method may be particularly useful in the epidemiological context of COVID-19 and other situations which require distant learning.

(Rev Med Chile 2021; 149: 989-996)

Key words: Computer Systems; Deep Learning; Internet-Based Intervention; Simulation Training; Social Media; Teacher Training.
'Servicio de Hematología, Hospital Regional Libertador Bernardo O'Higgins. Rancagua, Chile.

Escuela de Medicina, Universidad Mayor. Temuco, Chile. ${ }^{3}$ Departamento de Odontopediatría y Ortodoncia, Universidad de La Frontera. Temuco. Chile.

aPrograma de Magister en Innovación de la Docencia Universitaria en Ciencias de la Salud, Universidad de La Frontera.

${ }^{b}$ Doctor en Ciencias Médicas. 'Mg en Educación, Universidad Metropolitana en Ciencias de la Educación.

Fuente de apoyo financiero: El Dr. Carlos Flores Angulo recibió una beca de la Agencia Chilena de Cooperación Internacional para el Desarrollo del Ministerio de Relaciones Exteriores del Gobierno de Chile para cursar estudios de postgrado en la Universidad de La Frontera. Los autores declaran no tener conflictos de interés.

Recibido el 29 de diciembre de 2020, aceptado el 28 de abril de 2021.

Correspondencia a:

Paulo Sandoval Vidal

Avda Francisco Salazar 01145, Temuco;

paulo.sandoval@ufrontera.cl
E 1 modelo pedagógico aula invertida (AI) se caracteriza por ser un método activo en el cual hay un cambio de la estructura de curso, en este, el contenido es impartido antes de la clase y el tiempo destinado para ella se dedica a la resolución de problemas, profundizar en conceptos y fomentar el aprendizaje colaborativo, así el docente destina mayor tiempo para interactuar con los estudiantes ${ }^{1}$.

El AI se desarrolla en dos fases, la primera es asincrónica, en la cual los estudiantes se preparan para el tema que será tratado en la fase dos; para ello, el docente debe suministrar con antelación el recurso instruccional del contenido, lo que permite que los educandos experimenten todas las etapas del proceso de aprendizaje y reflexionen en su propio espacio y tiempo ${ }^{2}$. La segunda fase corresponde a un encuentro presencial, donde se aborda el tema desarrollado durante el tiempo extra-aula con la intención de alcanzar objetivos de aprendizaje más complejos ${ }^{3}$. 
La disponibilidad de herramientas de la Web 2.0, como Edpuzzle y Quizizz, dan la posibilidad que metodologías como el AI puedan ser adaptadas a una modalidad a distancia conservando sus características.

En este orden de ideas, la plataforma Edpuzzle puede permitir la ejecución de la primera fase del AI a través de vídeos educativos. Dicho programa ofrece diversas ventajas: es gratis (su versión básica), le ofrece al docente un registro de la visualización de la clase (incluso el número de veces que se ha repetido un segmento del vídeo), permite introducir preguntas (abiertas y cerradas) y notas de voz en el vídeo, así como retroalimentar a los estudiantes ${ }^{4,5}$. Existen datos que han demostrado que los estudiantes de medicina prefieren las vídeo-clases y selección de textos en el AI, siendo un recurso que suministra las herramientas necesarias para su participación activa ${ }^{6}$.

Para la ejecución de la segunda fase, es posible emplear el programa Quizizz que permite formular interrogantes y recibir las respuestas en tiempo real, siendo necesario que los participantes se encuentren conectados a internet empleando cualquier dispositivo electrónico. Esta herramienta puede ser utilizada en el aprendizaje basado en casos (ABC), brindando la posibilidad de vincular la teoría con la práctica. Al discutir un caso clínico relacionado con el tema impartido (Fase 1), los estudiantes evalúan su propia comprensión utilizando un alto orden de cognición, este proceso fomenta el aprendizaje activo y genera un resultado más productivo ${ }^{7}$.

Cuando este tipo de tecnologías se utilizan de forma apropiada se convierte en una herramienta mediática en pro del aprendizaje, donde docentes y estudiantes pueden interactuar, intercambiar ideas, consultar y producir conocimiento ${ }^{8}$.

Datos obtenidos de un metaanálisis de 26 artículos, determinó que los estudiantes de medicina de asignaturas preclínicas y clínicas tienen una buena percepción del $\mathrm{AI}^{9}$; encontrándose esta metodología asociada a mejores resultados académicos, así como al ejercicio y desarrollo de competencias durante el tiempo de clase.

Aunque el AI contempla una fase presencial, esta puede ser adaptada a una modalidad a distancia. Sin embargo, el primer paso para su posterior implementación corresponde a la evaluación de la percepción de los estudiantes, ya que, esta permite comprender, explicar e incluso predecir en gran medida el aprendizaje, y provocar en el enseñante la reflexión y mejora de su propia práctica docente.

Poder aplicar esta metodología utilizando herramientas de la Web 2.0, tiene implicaciones positivas en diversos escenarios como aquellos donde el desplazamiento a espacios físicos se encuentre limitado, incluyendo: a) Ubicaciones geográficas diferentes de los estudiantes y docentes; b) Interrupción no planificada de clases presenciales, y c) Situaciones epidemiológicas complejas como la pandemia por el SARS-CoV-2, cuya medida más recomendada para reducir su propagación ha sido el aislamiento social.

Por tales motivos, el objetivo del presente trabajo es describir la percepción en una muestra de estudiantes de medicina de la Universidad Mayor respecto al uso de herramientas de la Web 2.0 en la metodología AI.

\section{Material y Método}

\section{Población y muestra}

La población se encontró compuesta por 48 estudiantes de tercer año de Medicina de la Universidad Mayor que cursaron la asignatura "Integrado de Patología Morfofuncional” durante 2019. Solo aceptaron participar 39 estudiantes, de los cuales se obtuvo su consentimiento libre e informado, y se explicó la confidencialidad de los resultados.

\section{Metodología aula invertida}

Para la Fase 1 se elaboró el recurso instruccional digital (vídeo-clase) siguiendo las recomendaciones del modelo ADDIE ${ }^{10}$, el cual contempló las siguientes etapas:

Análisis: Se digitalizó parte del contenido especificado en el programa, los temas escogidos fueron: 1) Metabolismo del hierro; 2) Anemia por déficit de hierro; 3) Metabolismo de la vitamina B12 y ácido fólico y 4) Anemia megaloblástica.

Diseño: Los temas seleccionados fueron digitalizados y las vídeo-clases se encontraron disponibles en internet para su visualización asincrónica. Posteriormente, las dudas de los temas fueron discutidos en la segunda fase de la metodología.

Desarrollo: La virtualización de la clase se llevó a cabo empleando el programa Microsoft PowerPoint, para ello se grabó la explicación de 
Web 2.0 en el aula invertida - C. Flores-Angulo et al

cada diapositiva y posteriormente se guardó en formato mp4, la duración fue de 7 a $30 \mathrm{~min}$. En los vídeos se introdujeron preguntas con la intención de fomentar la interacción de los participantes y el trabajo colaborativo, permitiendo fijar aspectos claves del tema ${ }^{11}$.

Implementación: Se utilizó la plataforma de vídeos educativos Edpuzzle (https://edpuzzle. $\mathrm{com} /$ ). Para el ingreso, los estudiantes se debían registrar previamente y solo podían acceder al material empleando su usuario y contraseña. Las preguntas insertadas en las vídeo-clases debían ser respondidas para poder continuar con la visualización; además, la plataforma brinda la posibilidad de pausar el vídeo y guardar el avance, ya que, si el docente lo configura no será posible adelantar la vídeo-clase.

Evaluación: Las vídeo-clases (fase 1) y discusión (fase 2) fueron evaluadas mediante una encuesta compuesta por 13 ítems, previamente validada en español ${ }^{12}$ (Tabla 1). Los ítems del 1 al 12 fueron de tipo cerrados y se evaluaron empleando una escala de psicométrica del 1 al 8 (Desacuerdo: 1-3; De acuerdo: 4-6, Muy de acuerdo: 7-8); el ítem 13 fue una pregunta abierta (¿Qué mejoraría usted de la acción formativa desarrollada?). En dicha encuesta se indagaron aspectos relacionados a: presentación de la información en los vídeos, comunicación del tema, materiales audiovisuales e infraestructura.

En relación a la Fase 2 del AI, el contenido fue discutido en el aula de clases empleando la metodología de $\mathrm{ABC}$, para lo cual se elaboraron 22 casos clínicos del tema abordado, que fueron formulados empleando Quizizz (https://quizizz. $\mathrm{com} /$ ). La ejecución de este programa permitió evaluar en tiempo real las diferentes preguntas, resolver dudas y profundizar en la fisiopatología.

\section{Análisis de los datos}

Se empleó el programa estadístico SPSS versión $24\left(\right.$ IBM $^{\circledR}$, Armonk, NY, USA). La distribución de frecuencia fue expresada en porcentajes.

Se evaluó la consistencia interna de la encuesta mediante el estadístico alfa de Cronbach, considerándose aceptable un valor entre 0,80 a 0,90 , además, se determinaron los ítems que tienen menor consistencia interna y mayor redundancia para considerar su eliminación.

Las respuestas de la pregunta abierta fueron analizadas en base al contenido semántico, a través de la clasificación, codificación, categorización y saturación de la información empleando el programa ATLAS.ti versión 8.4. Posteriormente, las categorías y subcategorías fueron trianguladas por tres investigadores.

Tabla 1. Encuesta para la evaluación de la metodología aula invertida

Ítems
1. El tema tratado en la acción formativa es relevante para mi futura vida profesional
2. El tema tratado se presentó de forma interesante en los vídeos
3. La discusión en el salón de clase del tema tratado fue efectiva
4. El docente fue un comunicador eficaz
5. El docente estaba bien preparado
6. Los materiales audiovisuales fueron efectivos
7. El contenido discutido me será de utilidad a corto plazo
8. Podré aplicar mucho el contenido en mi futuro profesional
9. La infraestructura fue apropiada
10. El plan de trabajo fue correcto
11. Hubo un buen equilibrio entre las exposiciones y la participación del grupo
12. Creo que la acción formativa me ayudará en mi preparación profesional
13. ¿Qué mejoraría usted de la acción formativa desarrollada?




\section{Resultados}

\section{Consistencia interna del instrumento de recolección de datos}

La consistencia interna de la encuesta fue evaluada a partir de las respuestas suministradas por los 39 participantes, se determinó un valor de alfa de Cronbach de 0,881 , no encontrándose afectado por la supresión de algún ítem.

\section{Percepción de los estudiantes a la metodología aula invertida}

La encuesta fue respondida por $81,25 \%$, la distribución de frecuencia de cada ítem cerrado se encuentra descrita en la Tabla 2.

Respecto a los enunciados que hicieron referencia a la importancia del tema tratado (Ítems 1 al 4), se evidenció que una proporción pequeña de estudiantes, solo el 8\%, indicó que la Unidad de Hematología no le será útil en su vida profesional.

En relación a la evaluación general del AI, el 95\% y $98 \%$ de estudiantes estuvieron de acuerdo y muy de acuerdo con la infraestructura (Ítem 5) y con el plan de trabajo (Ítem 6), respectivamente.

La opinión de los estudiantes de la Fase 1 del método, que contempló el uso de vídeo-clases, indicó que el 69\% de los estudiantes estuvieron muy de acuerdo en la forma como se presentó el tema (Ítem 7) y en la efectividad de los recursos audiovisuales (Ítem 8).

$\mathrm{Al}$ analizar la opinión de la fase 2 , que contempló la discusión de casos clínicos, el 3-5\% de los participantes no estuvieron de acuerdo con la discusión (Ítem 9) y con el equilibrio entre la clase y la participación del grupo (Ítem 10), evidenciándose en este último punto que solo el $38 \%$ estuvo muy de acuerdo con el equilibrio entre ambas fases.

Por otro lado, el $87 \%$ y $100 \%$ de los encuestados tuvieron la percepción que el docente fue un comunicador eficaz (Îtem 11) y se encontraba bien preparado (Ítem 12).

\section{Opinión de los estudiantes respecto a la acción formativa}

En la Figura 1 se encuentran representadas las categorías de los comentarios a la pregunta: ¿Qué mejoraría usted de la acción formativa desarrollada? De los 39 estudiantes que respondieron la encuesta se identificaron 44 tipos de comentarios que se agruparon en 6 categorías.

La categoría más frecuente contenía comentarios que indicaron buena percepción a la metodología, donde destacaron: "La verdad me encanto esta metodología, me quedo todo muy claro, si

Tabla 2. Distribución de frecuencia del nivel de satifacción de la intervención educativa

\begin{tabular}{|c|c|c|c|c|c|c|}
\hline \multirow[b]{2}{*}{ Ítem } & \multicolumn{2}{|c|}{ Desacuerdo } & \multicolumn{2}{|c|}{ De acuerdo } & \multicolumn{2}{|c|}{$\begin{array}{l}\text { Muy de } \\
\text { acuerdo }\end{array}$} \\
\hline & $\mathbf{n}$ & $\%$ & $\mathbf{n}$ & $\%$ & $\mathbf{n}$ & $\%$ \\
\hline $\begin{array}{l}\text { 1. El tema tratado en la acción formativa es relevante para mi futura } \\
\text { vida profesional }\end{array}$ & 0 & 0 & 2 & 5 & 37 & 95 \\
\hline 2. El contenido discutido me será de utilidad a corto plazo & 3 & 8 & 10 & 26 & 26 & 67 \\
\hline 3. Podré aplicar mucho el contenido en mi futuro profesional & 1 & 3 & 7 & 18 & 31 & 79 \\
\hline 4. Creo que la acción formativa me ayudará en mi preparación profesional & 1 & 3 & 3 & 8 & 35 & 90 \\
\hline 5. La infraestructura fue apropiada & 2 & 5 & 5 & 13 & 32 & 82 \\
\hline 6. El plan de trabajo fue correcto & 1 & 3 & 12 & 31 & 26 & 67 \\
\hline 7. El tema tratado se presentó de forma interesante en los vídeos & 1 & 3 & 11 & 28 & 27 & 69 \\
\hline 8. Los materiales audiovisuales fueron efectivos & 2 & 5 & 10 & 26 & 27 & 69 \\
\hline 9. La discusión en el salón de clase del tema tratado fue efectiva & 1 & 3 & 14 & 36 & 24 & 62 \\
\hline $\begin{array}{l}\text { 10. Hubo un buen equilibrio entre las exposiciones y la participación del } \\
\text { grupo }\end{array}$ & 2 & 5 & 22 & 56 & 15 & 38 \\
\hline 11. El docente fue un comunicador eficaz & 0 & 0 & 5 & 13 & 34 & 87 \\
\hline 12. El docente estaba bien preparado & 0 & 0 & 0 & 0 & 39 & 100 \\
\hline
\end{tabular}


A)

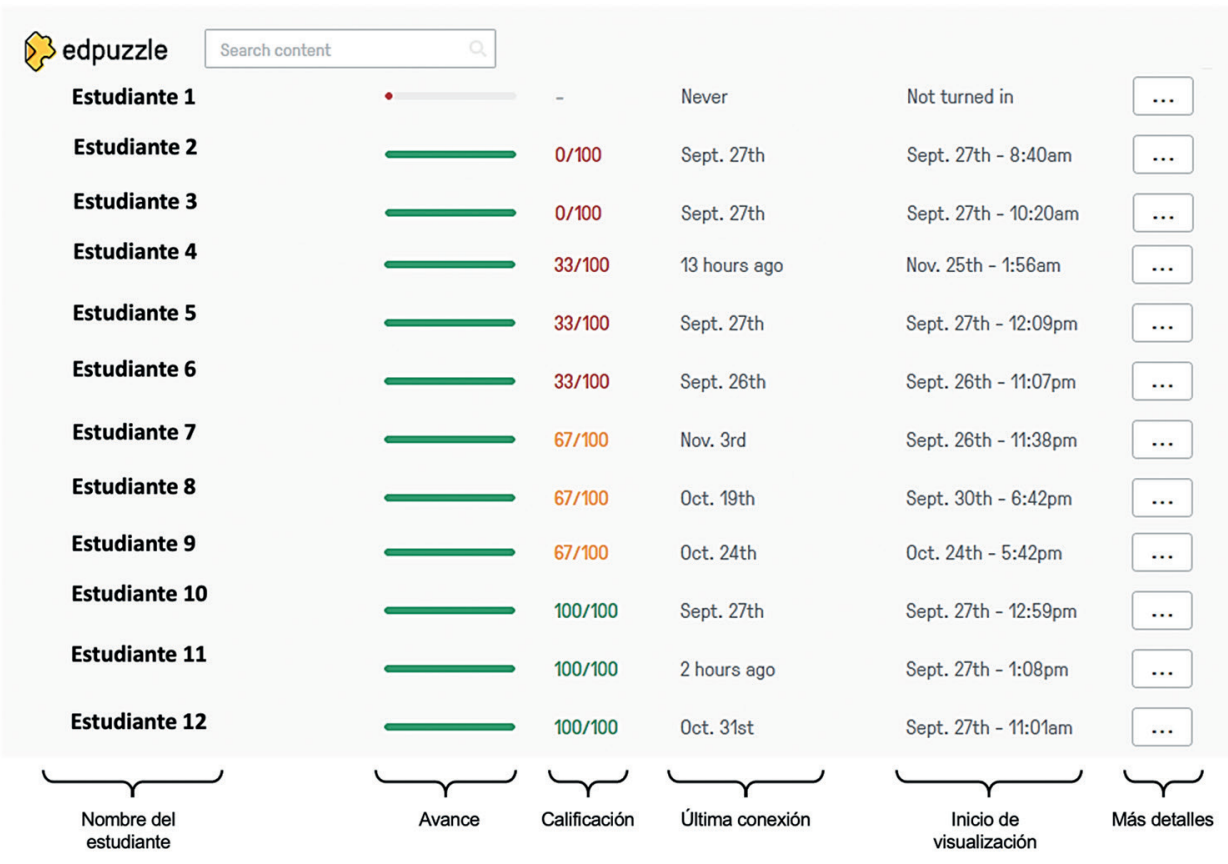

B)

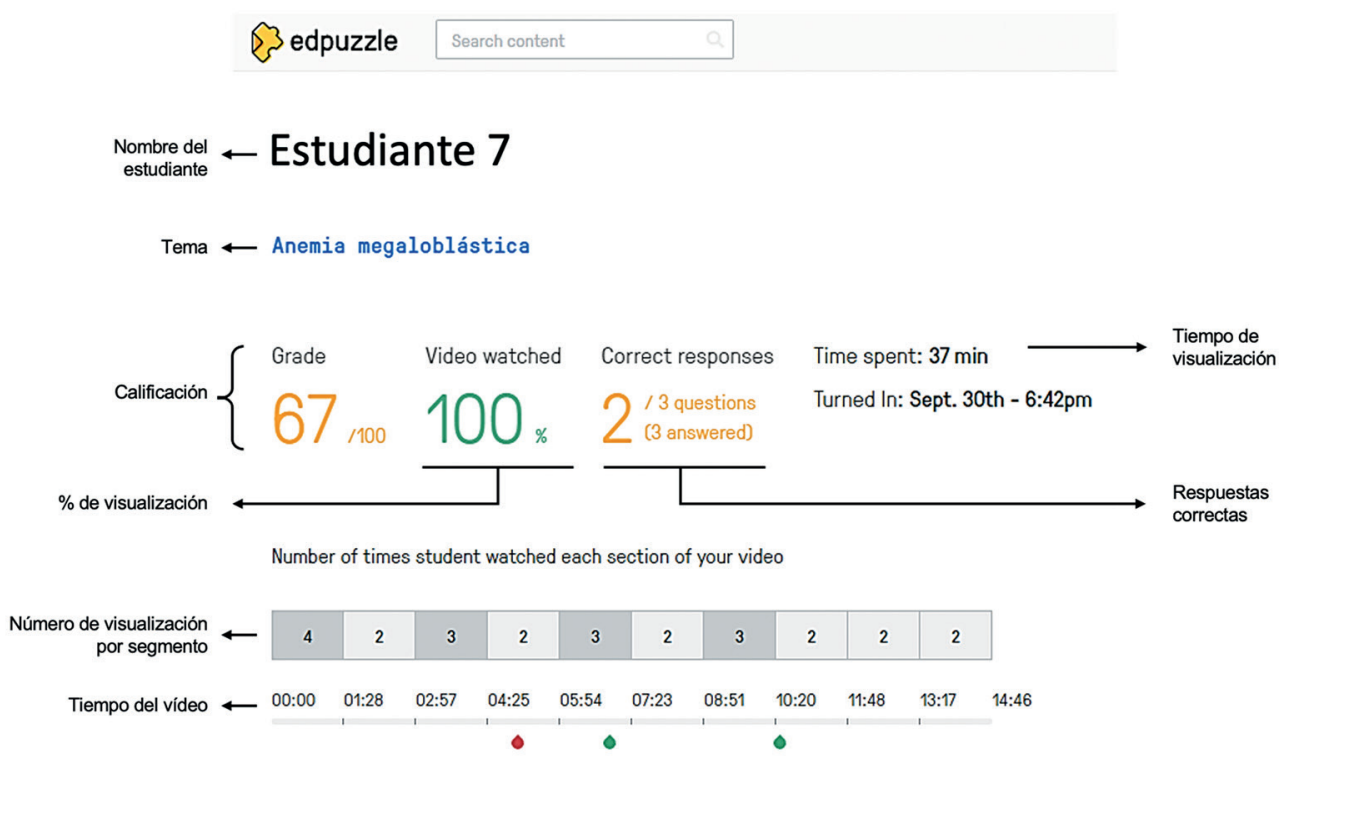

Figura 1. Reporte de datos obtenidos de Edpuzzle. A) Edpuzzle otorga información organizada por estudiante, donde se evidencia el avance en la vídeo-clase, la calificación obtenida en base a 100 punto, la última conexión del estudiante y el inicio de la visualización de la clase; B) En la parte inferior se muestra el número de veces que cada estudiante a visualizado segmentos específicos de la clase; también se muestran puntos de diferentes colores que corresponde a cada pregunta formulada (roja: respuesta incorrecta; verde: respuesta correcta). Edpuzzle informa el detalle de la respuesta de cada pregunta (dato no mostrado). 


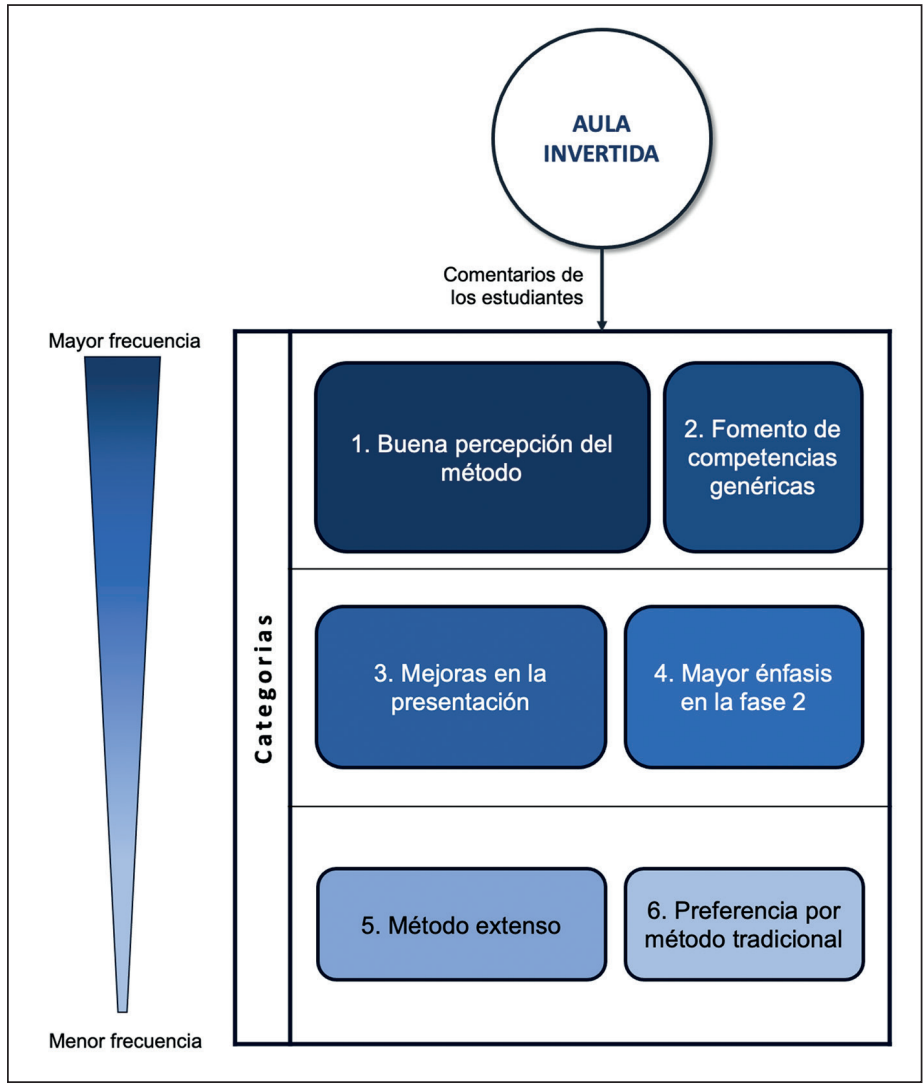

Figura 2. Categorias de respuestas obtenidas de la pregunta abierta: ¿Qué mejoraría usted de la acción formativa desarrollada? tenía dudas podía retroceder el vídeo, etc...”; “En general me pareció bastante acertado el método empleado para ser la primera vez que nuestro curso tiene este tipo de contacto con el sistema usado"; "La idea de hacer vídeo-clases y después comentarlas de manera presencial es excelente".

Le siguió en frecuencia, aquellas citas que mencionaron que, el método estimulaba el desarrollo de ciertas competencias genéricas que incluyeron: aprendizaje autónomo, habilidades de investigación, capacidad de adaptarse a nuevas situaciones, y capacidad para organizar y planificar el tiempo; sin embargo, a pesar de su identificación fueron valoradas de forma negativa, encontrándose comentarios como: “...tuve que buscar información desde diversas fuentes en Internet para captar las nociones básicas que no recordaba de años anteriores, creo que una inducción al tema podría haber sido muy provechosa...”; “...quisiera a alguien que pueda pararse frente a mí con la intención de querer informarme sobre algún tema..."; "Pre- fiero mucho más a un docente explicando en una sala de clases...”.

Algunos sugirieron la necesidad de mejoras puntuales en la presentación, como el tiempo, uso de recursos visuales más interactivos y dar mayor énfasis en la discusión y aplicación del tema. Un grupo menos numeroso comentó que la metodología requirió una gran inversión de tiempo. Hubo pocos alumnos, que prefieren continuar con el método tradicional y mencionaron aspectos como: "No considero personalmente una técnica que favorezca mi aprendizaje..."; “...no me gusta esta forma de aprendizaje, ya que es difícil concentrarme mirando un video...".

\section{Discusión}

En el presente estudio se obtuvo información respecto a la percepción del AI en una muestra por conveniencia de estudiantes de medicina. 
Aunque los resultados reflejaron la diversidad de opiniones de los estudiantes, se determinó que la mayoría estuvo muy de acuerdo con el método. La distribución de frecuencia fue similar a lo reportado por Angadi y cols. en 49 estudiantes de medicina de la Jawaharlal Nehru Medical College en la India $\left(\chi^{2}=2,512 ; p=0,2847\right)^{13}$.

Aunque diferentes grupos de investigación han demostrado que los estudiantes de ciencias de salud prefieren el método AI respecto al tradicional ${ }^{14}$, en la mayoría de las investigaciones se requirió un encuentro presencial.

La información obtenida de este estudio indicó que los estudiantes se sienten cómodos empleando plataformas educativas disponibles en internet, permitiendo que el AI pueda ser ejecutada a distancia. Sin embargo, una aspecto que debe ser mejorado corresponde al $A B C$, debido a que algunos manifestaron que no lograron aprovechar al máximo la sesión de discusión de casos clínicos; este segmento indicó que se debió a la poca experiencia con el método y la poca preparación previa del tema, donde destacaron comentarios como: "...es algo nuevo para todos nosotros y espero que en actividades futuras funcione cada vez mejor..."; "... Lamentablemente la mayor falencia se vio en la poca preparación del curso...”. Reportes similares fueron descritos por Whelan y cols. ${ }^{15}$ e Ilic $^{16}$, no obstante, se esperaría que mejore la situación al continuar con las sesiones y cuando los estudiantes noten el beneficio de la discusión presencial/sincrónica y la importancia de la revisión del material a discutir.

Respecto al recurso instruccional empleado para la preparación del AI, en el estudio se emplearon vídeo-clases pregrabadas, documentándose alto nivel de satisfacción en la muestra analizada, lo cual es concordante a lo reportado por Bouwmeester y cols. ${ }^{6}$; sin embargo, los estudiantes han sugerido la necesidad de incorporar mayores elementos interactivos. Para la ejecución de los vídeos se empleó Edpuzzle debido a las diversas ventajas que ofrece y por la existencia datos como los reportados por Silverajah \& Govindaraj, quienes realizaron un estudio en un curso de química en Malasia, el cual indicó que los estudiantes tuvieron una percepción positiva $(77,8 \%$ a $94,5 \%)$ respecto al uso de Edpuzzle y el desarrollo del aprendizaje autónomo ${ }^{17}$. En este orden de ideas, Kaplan \& Tüzer compararon en una muestra de estudiantes de enfermería (Universidad Ankara,
Turquía) la efectividad del aprendizaje basado en web (empleando Edpuzzle) con el aprendizaje entre pares, determinando que el uso de vídeo-clases fue igualmente efectivo ${ }^{18}$.

Respecto a la duración del método, aunque se emplearon video-clases bien concisas, a algunos estudiantes les pareció que el AI corresponde a un método extenso. En estudios previos, se demostró la preocupación de los estudiantes de medicina respecto a la cantidad de tiempo que se requiere para la preparación y análisis previo a la clase ${ }^{19}$. Por tales motivos, es importante considerar el tiempo total (visualización y discusión) para que los estudiantes no tengan una demanda excesiva de las asignaturas que emplean dicha metodología.

De acuerdo a lo reportado por Street y cols., los estudiantes de medicina sintieron que el AI ayudó al mejoramiento de ciertas competencias genéricas como el pensamiento analítico y resolución de problemas en comparación con el aula tradicional $^{20}$. En nuestro estudio, aunque se determinó que una proporción importante de estudiantes hicieron comentarios relacionados al fomento de competencias genéricas, una proporción importante de los comentarios (75\%) fueron negativos, lo cual, puede sugerir poca experiencia de las metodologías activas en asignaturas anteriores y mayor confort con los métodos pasivos. Algunos investigadores han descrito que los estudiantes prefieren que los docentes direccionen más el aprendizaje $\mathrm{e}^{15}$, no obstante, esto le restaría valor a los métodos centrados en el estudiante.

En conclusión, la ejecución del AI empleando plataformas disponibles en línea como Edpuzzle y Quizizz fue bien percibida por el grupo de estudiantes encuestados. Aunque es necesario realizar mejoras, se recomienda su uso en la pandemia del COVID-19 y otras situaciones epidemiológicas y sociales que compartan características similares.

Es necesario profundizar en esta línea de investigación de modo de obtener información de la percepción de los docentes respecto al nivel de logro de los resultados de aprendizaje y comparar con otras metodologías en asignaturas básicas y clínicas.

\section{Referencias}

1. DeLozier SJ, Rhodes MG. Flipped classrooms: a review of key ideas and recommendations for practice. Educ Psychol Rev 2017; 29(1): 141-51. 
2. Cavert MS. Why flip the medical resident classroom? A pilot study in Singapore. En: Walker Z, Tan D, Koh NK, Editores, Flipped classrooms with diverse learners. Singapore: Springer; 2020. p. 255-67.

3. Tolks D, Schäfer C, Raupach T, Kruse L, Sarikas A, Gerhardt-Szép S, et al. An Introduction to the Inverted/ Flipped Classroom model in education and advanced training in medicine and in the healthcare professions. GMS J Med Educ 2016; 33(3): Doc46.

4. Shelby SJ, Fralish Z. Using Edpuzzle as a method to improve student engagement and performance in the introductory biochemistry laboratory. FASEB J 2020; 34(S1): 1-1.

5. López GA, Álvarez C, Lerma JM, Carralero D. Elaboración de vídeos docentes: más allá de la Flipped Classroom. Hekademos 2020; (28): 17-23.

6. Bouwmeester RA, de Kleijn RA, ten Cate OTJ, van Rijen HV, Westerveld HE. How do medical students prepare for flipped classrooms?. Med Sci Educ 2016; 26(1): 53-60.

7. Gade S, Chari S. Case-based learning in endocrine physiology: an approach toward self-directed learning and the development of soft skills in medical students. Adv Physiol Educ 2013; 37(4): 356-60.

8. Hernández S. El modelo constructivista con las nuevas tecnologías: aplicado en el proceso de aprendizaje. Rev Univ Soc Conoc 2008; 5(2): 26-35.

9. Ramnanan CJ, Pound LD. Advances in medical education and practice: student perceptions of the flipped classroom. Adv Med Educ Pract 2017; 8: 63-73.

10. Minasian-Batmanian LC. Guidelines for developing an online learning strategy for your subject. Med Teach 2002; 24(6): 645-7.

11. Mischel LJ. Watch and Learn? Using EDpuzzle to enhance the use of online videos. Manag Teach Rev 2019; 4(3): 283-9.

12. Kirkpatrick D, Kirkpatrick J. Evaluación de acciones formativas: los cuatro niveles. Barcelona, España: Epise; 2007. p. 51-67

13. Angadi NB, Kavi A, Shetty K, Hashilkar NK. Effectiveness of flipped classroom as a teaching-learning method among undergraduate medical students - An interventional study. J Educ Health Promot 2019; 8: 211.

14. Hew KF, Lo CK. Flipped classroom improves student learning in health professions education: a meta-analysis. BMC Med Educ 2018; 18(1): 38.

15. Whelan A, Leddy JJ, Mindra S, Matthew Hughes JD, ElBialy S, Ramnanan CJ. Student perceptions of independent versus facilitated small group learning approaches to compressed medical anatomy education. Anat Sci Educ 2016; 9(1): 40-51.

16. Ilic D, Hart W, Fiddes P, Misso M, Villanueva E. Adopting a blended learning approach to teaching evidence based medicine: a mixed methods study. BMC Med Educ 2013; 13: 169.

17. Silverajah VG, Govindaraj A. The use of Edpuzzle to support low-achiever's development of self-regulated learning and their learning of chemistry. Proceedings of the 10th International Conference on Education Technology and Computers 2018: 259-63.

18. Kaplan S, Tüzer H. The effect of web-assisted learning and peer learning on the stoma care-related knowledge and skills of nursing students. Cukurova Med J 2020; 45(2): 604-12.

19. Evans KH, Thompson AC, O’Brien C, Bryant M, Basaviah $\mathrm{P}$, Prober $\mathrm{C}$, et al. An innovative blended preclinical curriculum in clinical epidemiology and biostatistics: impact on student satisfaction and performance. Acad Med 2016; 91(5): 696-700.

20. Street SE, Gilliland KO, McNeil C, Royal K. The flipped classroom improved medical student performance and satisfaction in a pre-clinical physiology course. Medical Science Educator 2015; 25(1): 35-43. 